\title{
Hemangiomas of Head and Neck - A Review
}

\author{
T.Rohini ${ }^{1}$, S.GideanArularasan ${ }^{2}$, M.Murugan ${ }^{3}$, \\ ${ }^{1}$ (Department Of Maxillofacial Surgery/Tamilnadu Government Dental College And Hospital/Chennai) \\ ${ }^{2}$ (Dental/Christian Medical College And Hospital,Vellore) \\ ${ }^{3}$ (Oral And Maxillofacial Surgeon,Madurai)
}

\begin{abstract}
Hemangiomas which are tumors of vascular origin constitute $7 \%$ of all benign tumours. Hemangiomas of the head and neck region comprise about $60-70 \%$.. Hemangiomas can be infantile or congenital Hemangiomas. Infantile Hemangiomas $(I H)$ are more common in pre mature infants. Congenital Hemangiomas are tumors that occur fully grown at birth and do not manifest the postnatal course and lifecycle of common IH. They can be sub classified as rapidly involuting congenital Hemangiomas(RICH) and Non Involuting congenital Hemangiomas(NICH). The purpose of this article is to give a comprehensive review of hemangiomas and the diverse treatment options available for this complex endothelial tumour and to stress the fact of individualizing a treatment protocol.
\end{abstract}

Keywords: ,congenital hemangioma,infantile hemangioma

\section{I . Introduction}

Hemangiomas which are tumors of vascular origin constitute $7 \%$ of all benign tumours ${ }^{1}$. Hemangiomas of the head and neck region comprise about $60-70 \%{ }^{2}$. According to Enzinger and Weiss, Hemangiomas are broadly classified into Capillary, Cavernous and miscellaneous forms like Verrucous, Venous, Arterio venous Hemangiomas and so forth ${ }^{3}$. Hemangiomas can be infantile or congenital Hemangiomas. Infantile Hemangiomas(IH) are more common in pre mature infants with a reported incidence of $23 \%$ in neonates weighing less than 1200 grams $^{4}$. It is also the most common tumors of infancy and childhood occuring in 4- 10\% of Caucasian infants 5 . In contrast congenital Hemangiomas are tumors that occur fully grown at birth and do not manifest the postnatal course and lifecycle of common IH. They can be sub classified as rapidly involuting congenital Hemangiomas(RICH) and Non Involuting congenital Hemangiomas(NICH).

\section{Infantile hemangiomas}

Infantile Hemangiomas grow rapidly, regress slowly and never recur. The three stages in the lifecycle of a Hemangioma are proliferating phase (0-1 yrs of age), involuting phase (1-5 yrs of age) and involuted phase ( $>5$ yrs of age). The three stages can be distinguished clinically, microscopically and immunohistochemically ${ }^{6}$ Histologically in the proliferating phase, there are plump rapidly dividing endothelial cells forming tightly packed sinusoidal channels, whereas in the involuting phase the endothelial proliferation is decreased, apoptosis is increased and fibro fatty replacement of Hemangiomas has just begun ${ }^{8}$. During the involuted phase all that remains are few tiny capillary like feeding vessels and draining veins surrounded by islands of fibro fatty tissue admixed with dense collagen and reticular fibres.

Immunohistochemically angiogenic proteins such as fibroblast growth factor [bFGF] and vascular endothelial growth factor [VEGF] and enzymes involved in remodeling of extra cellular matrix are prominent in proliferating hemangiomas and diminishes to normal level during regression? Clinically if the tumor is in the superficial dermis the skin is raised, crimson in color and firm and rubbery to palpation. If the tumor is in the deeper dermis and sub cutis, the lesion appear raised with a bluish hue. As it regresses it becomes softer and erythematous color changes to a grey hue. After involution is complete there may be redundant atrophic skin, yellow discoloration or scarred patches in instances of previous ulceration, residual fibro fatty tissue and telangiectasia?

Multi focal Hemangiomas involving visceral organs, if large can divert significant intra vascular volume leading to high output cardiac failure ${ }^{10}$. A dermatomal distribution in V1 ,V2,V3 should rule out PHACES Syndrome (Posterior fossa malformations, Hemangiomas arterial anomalies, coarctation of aorta, cardiac defects and eye abnormalities) $)^{11}$. Periorbital hemangiomas can block the visual axis causing deprivation ambylopia or may extend into retro bulbar space leading to ocular proptosis ${ }^{12}$. Subglottic Hemangiomas are life threatening lesion presenting with symptoms 
such as hoarseness, biphasic stridor and airway obstruction ${ }^{13}$. Ulceration occur in $5 \%$ of cutaneous Hemangiomas and are more common in lesion involving lip, perineum, anogenital area and extremities ${ }^{14}$.

\section{Congenital hemangiomas}

Rapidly involuting Hemangiomas are more common in the trunk, head or neck region. Three morphologic variants of RICH exist,

a) A lesion with characteristic red purple color with a coarse telangiectasia.

b) A flat infiltrative tumor with violaceous overlying skin.

c) Raised grayish tumor with multiple tiny telangiectasia with pale halo ${ }^{15}$

Histologically RICH is composed small to large lobules of capillaries with moderately plump endothelial cells and pericytes.NICH are also more common in neck, trunk or limbs. They tend to be plaque like with a pink to purple color with prominent coarse telangiectasia. Lesion are warmer to palpation than the surrounding skin. Histopathology reveals lobular collections of small thin walled vessels with large stellate shaped central vessels ${ }^{17}$.

\section{Pathogenesis of hemangiomas:}

The placental theory for the origin of Hemangiomas was proposed by North et $\mathrm{al}^{18}$ who reported that infantile Hemangiomas may originate from either invading angioblasts that differentiate towards a placental phenotype or from embolised placental cells. GLUT 1 is expressed throughout the three phases of infantile Hemangiomas making it a valuable tool in diagnosis of infantile Hemangiomas. On the contrary both RICH and NICH are GLUT 1 negative ${ }^{19}$. Dadras et al showed that lymphatic endothelial hyaluronan receptor LYVE -1 a marker was strongly expressed in proliferating Hemangiomas, diminished in involuting and absent in involuted Hemangiomas $^{20,21,22}$.

\section{Imaging}

The key imaging required for the diagnosis of Hemangiomas are ultra sound, doppler ultra sound and MRI. CT fails to adequately delineate soft tissue planes. MRI produces high signal intensity representing blood as well as focal heterogeneities representing areas of fibrosis, thrombosis or calcification. Doppler shows arterial or venous waveforms with high vessel density $>5$ vessels / $\mathrm{cm}^{2}$ with high doppler shift $>2 \mathrm{kHz}^{23}$. A study detailing the imaging tendencies of congenital vs infantile Hemangiomas reported the following including hetrogeneity $(72 \%$ of $\mathrm{NICH} \quad, \quad 62.5 \%$ of $\mathrm{RICH}$ vs $92.31 \%$ of infantile Hemangiomas), visible vessels (72\% NICH, $62.5 \%$ RICH vs $15.4 \%$ of infantile Hemangiomas) and calcification (17\% NICH, $37.5 \%$ RICH vs none in infantile Hemangiomas $)^{24,25}$. Angiography of RICH demonstrates a well circumscribed areas with an intense tissue staining in a lobular pattern with enlarged surrounding systemic artery branches ${ }^{26}$.

\section{VI.Management}

The treatment of choice for Hemangiomas depend primarily on the site and growth stage of the lesion $^{27}$. The rationale behind treating a Hemangioma are to prevent or improve functional impairment or pain, to prevent or improve scarring or disfigurement or to avoid life threatening complications. Small, isolated or multiple skin lesions on face in infants are treated early to avoid progression into proliferation. Imiquimod is an immuno modifier used for small and intermediate sized Hemangiomas with alternate day topical application for a cycle of 3-5 months $^{28}$. The drawback of the drug is hyper pigmentation which makes it use in the highly esthetic region such as the face controversial.

Laser therapy is indicated in cases of superficial proliferating Hemangiomas. The laser therapy accelerates the regression or reduces the sizes of the lesion. The choice of laser depends on location, depth and size of the lesion.

Flash lamp pumped pulsed dye laser with a wavelength of 585 or $595 \mathrm{~mm}$ is the only laser that photo coagulates the target vessels leaving the overlying skin intact thereby making it useful for superficial Hemangiomas or those at involution stage, however it is of little use in deep seated Hemangiomas because of limited penetration depth $^{29}$. Neodymium Yttrium aluminum garnet laser (Nd:YAG) having wavelength of $1064 \mathrm{~nm}$ and penetration depth of $5 \mathrm{~mm}$ is the choice for larger or $2 \mathrm{~cm}$ deep seated Hemangiomas. Percutaneous laser therapy can be used for deep Hemangiomas but cooling devices are needed to lower the temperature to prevent damage to the 
epidermis from thermal injury ${ }^{30}$. The effectiveness of laser is $77-100 \%$ in treating Hemangiomas.

Drug therapy is indicated for mixed proliferative and Hemangiomas affecting vital organs or life threatening Hemangiomas. Oral prednisolone is more effective than intravenous injection of methyl prednisolone. The effectiveness rate is $84 \%$ with a significant relationship between dosage and effectiveness ${ }^{31}$. The regimen for oral corticosteroid is oral predinisolone (3-5 $\left.\mathrm{mg} / \mathrm{kg}\right)$ every morning for 6-8 wks. The dose is tapered after that for 2 or 3 wks. The treatment can be repeated for 2 or 3 cycles at an interval of $4-6 \mathrm{wks}^{32}$. In case of more localized Hemangiomas such as orbital or parotid lesion, intra lesional steroid triamcinocolone acetonide $1-2 \mathrm{mg} / \mathrm{kg}$ body weight is given at monthly intervals ${ }^{33}$

Pingyangmycin (bleomycin A5) having a high scelerosing effect on vascular endothelium is found to have greater than $90 \%$ success rate and $49 \%$ complete resolution ${ }^{31}$. It is suitable for proliferating Hemangiomas responding poorly to steroids or laser therapy, for cutaneous superficial or mucosal Hemangiomas .Its concentration is $1 \mathrm{mg} / \mathrm{ml}$; for subcutaneous or deep, the concentration is $1.5-2 \mathrm{mg} / \mathrm{ml}^{34,35}$. In case of Hemangiomas unresponsive to steroids or recurrence after steroids, Vincristine $0.5-1 \mathrm{mg} / \mathrm{ml}$ can be given intravenously once a week over 6 weeks $\mathrm{s}^{36}$. In case of life threatening platelet consumptive coagulopathy although more common in the trunk than the head and neck, diluted ethanol embolo therapy is reported to be effective. Alphainterferon has been effective but has serious side effects such as spastic diplegia ${ }^{37}$.

Recently propanolol has been used to treat infantile Hemangiomas on the basis that propanolol being a $\beta$ - blocker induce apoptosis by antogonising GLUT-1 receptors . Leslie et al reported a protocol for usage of propanolol. Starting dose of $0.17 \mathrm{mg} / \mathrm{kg}$ given at 8 hourly intervals. vitals signs and blood glucose are monitored one hour after each dose. if the first two doses are tolerated, the amount is doubled to $.33 \mathrm{mg} / \mathrm{kg} / \mathrm{dose}$. after two more doses, the amount is doubled to $0.67 \mathrm{mg} / \mathrm{kg} / \mathrm{dose}$. for infants less than 3 months of age, slower dose escalation is recommended to avoid the risk of hypoglycaemia ${ }^{38}$.

The choice of a surgical resection for Hemangiomas should be made taking into account that surgical intervention would be more esthetically acceptable than that from the medical treatment or from observation. The surgical indications for proliferating Hemangiomas are,

a) Hemangiomas located in the nose and lip that did not respond to other treatment.

b) eyelids that cause visual interference.

c) repeated bleeding from the Hemangiomas ${ }^{39}$.

Residual deformities often require surgical recontouring to improve esthetics and function. Local wound care is needed to alleviate pain and infection in ulcerated lesion. Debridement of the ulcer in conjunction with topical mupirocin, bacitracin or metronidazole is needed.

\section{Conclusion}

Hemangiomas are a complex group of endothelial tumor having a heterogenicity in their presentation and treatment of Hemangiomas have always been a controversial issue, any treatment decision whether surgical or medical must be critically evaluated in terms of cure and esthetic results,and the choice of treatment plan should be individualized rather than a fixed treatment protocol.

\section{References}

[1]. Tarkan. Cavernous Hemangiomas of temporalis muscle: report of a case and review of the literature: Turk Neurosurg 2007; 17 : 33-36.

[2]. K. A. Kamala, L. Ashok,and G. P. Sujatha Cavernous Hemangiomas of tongue : a rarecase report : Contemp Clin Dent. 2014; 5: 95-98.

[3]. F.M Enzinger \& S.W Weiss : Soft tissue tumors Mosby, St louis Mo, USA, 5th edition 2001.

[4]. Amir .J .Strawberry Hemangiomas in pre term infants. pediatr Dermato . 1986;1 3 :331-332.

[5]. Marter JJ, Mulliken JB : Current management of Hemangiomas and vascular

[6]. malformation, clin plast surg 2005;32:99-116.

[7]. Mulliken JB, Enjolras O . Congenital Hemangiomas and Infantile Hemangiomas : missing links. J Am Acad Dermatal.2004; 50: 875-882.

[8]. Takahashi K : Cellular Markers that distinguish the phases of Hemangiomas of infancy and childhood, J clin Invest . 1994;93 : 2357-2364.

[9]. Gonzalez -Crussi.F Reyes - Mugica M. Cellular Hemangiomas in infants : light microscopic, immunohistochemical and ultra structural observation. Am J Surgery Path.1991; $15: 769$ - 778.

[10]. Marler JJ, Fishman SJ, Kilroy SM, Fang J, Upton J, Mulliken JB, Burrows

[11]. PE,Zurakowski D, Folkman J, Moses MA. Increased expression of urinary matrix metallo proteinase parallels the extent and activity of vascular anomalies. Pediatrics.2005; $116: 38-45$.

[12]. Boon LM,Burrows PE, Paltiel HJ, Lund DP, Ezekowitz RA, Folkman J, Mulliken JB. Hepatic vascular anomalies in infancy : a twenty seven years experience. J Pediatr.2006; 1129: 346-354.

[13]. Frieden, I.J.,Reese,V., Cohen, D.PHACE syndrome. Arch Dermatol. 1996;132:307-311 
[14]. Elsas FJ, Lewis AR. Topical treatment of periocular capillary hemangioma. J Pediatr Ophthalmol .1994;31:153-6.

[15]. Orlow SJ, Isakoff MS, Blei F. Increased risk of symptomatic hemangiomas of the airway in association with cutaneous hemangiomas in a "beard" distribution.J Pediatr 1997;131:643- 6.

[16]. 14. Jennifer J. Marler, John B. Mulliken-Current management of hemangiomas and vascular malformations.Clin Plastic Surg.2005; $32: 99-116$

[17]. Alfons Krol,CarolJ,.MacArthur,.congenitalhemangiomas:rapidlyinvolutingand non involutinghemangiomas..Arch Facial Plast Surg. 2005; 7 : 307-311.

[18]. BerenguerB, Mulliken JB, Enjolras O, Boon LM,Wassef M, Josset P, Burrows PE, Perez-Atayde AR,KozakewichHP.Rapidly nvoluting congenital Hemangiomas :

[19]. Clinical and Histo Pathologic feature : Pediatic dev Pathol. $2003 ; 6$ : 495-510

[20]. Enjolras O, Mulliken JB, Boon LM, et al. Noninvoluting hemangioma: a rare cutaneous vascular anomaly. Plast Recon Surg 2001;107:1647 - 54

[21]. Paula E North, Milton Waner, Michael C Brodsky : Are infantile hemangiomas of placental origin ? ophthamology , 2002; 109 : 633-634

[22]. Paula E. North,Milton Waner, Adam Mizeracki,, Martin C. Mihm Jr.A newly discovered immunohistochemical marker for Juvenile hemangiomas : Hum Pathol 2000; 31 : 11-22.

[23]. adrasSS,North PE,BertonciniJ, Mihm MC, Detmar M. Infantile Hemangiomas are arrested in an early developmental vascular differentiation state : Mod Pathol: 2004; 17 :1068-1079.

[24]. Lisa H. Lowe, Tracy C. Marchant, Douglas C. Rivard, and Amanda J. Scherbel,: Vascular malformation : Classification and terminology the radiologist needs to know : Seminarin Roentgenology. Elsevier Inc.2012; 106-117

[25]. Rogers M, Lam A, Fischer G: Sonographic findings in series of (RICH). PediatrDermatol. 2002;19:5. -11.

[26]. Konez O, Burrows PE, Mulliken JB, et al: Angiographic features of rapidly involutingcongenital hemangioma (RICH). Pediatr Radiol.2003; 33:15-19.

[27]. Zheng JW, Zhou GY, Wang YA, Zhang ZY.Management of head and neck hemangiomas in China.Chin Med J (Engl) 2008;121:1037-42

[28]. Mc Cuaig CC1, Dubois J, Powell J, Belleville C, David M, Rousseau E, Gendron R, Jafarian F, Auger I. : A phase II, open label study of the efficiency and safety of imiquimod in the treatment of mixed and infantile hemangiomas : Pediatr dermatol, $2009 ; 26$ : 203-212.

[29]. Michel JL. Treatment of hemangiomas with $595 \mathrm{~nm}$ pulsed dye laser dermobeam.Eur J Dermatol. 2003;13(2):136-141.

[30]. Vesnaver A, Dovsak DA. Treatment of vascular lesions in the head and neck using Nd: YAG laser. JCraniomaxillofac Surg. 2006;34:17-24

[31]. Bennett ML, Fleischer AB Jr, Chamlin SL, Frieden IJ : Oral Corticosteriod use is effective for cutaneous hemangiomas: an evidence based evaluation : Arch Dermatol. 2001; 137: 1208 - 1213.

[32]. Heng, J.W., Yang, X.J., Zhou, Q., Wang, Y.A., Ye, W.M., Zhu, H.G. et al. Short-term high-dose oral prednisone on alternate days: a more rational regime for infantile hemangiomas.Med Hypotheses. 2009; 72: 365

[33]. A.N. Gangopadhyay, S.P. Sharma, S.C. Gopal, D.K. Gupta, K. Panjawani and J.K. Sinha : Local Steriod therapy in cutaneous hemangiomas. Indian Pediatr 1996;33:31-3

[34]. Muir T, Kirsten M, Fourie P, Dippennar N, Ionescu GO. Interalesional bleomycininjection(IBI) treatment for haemangiomas and congenital vascular malformations.PediatrSurg Int 2004;19:766-73.

[35]. Omidvari S, Nezakatgoo N, Ahmadloo N, Mohammadianpanah M, Mosalaei A : Role of intralesional bleomycin in the treatment of complicated hemangiomas : a prospective clinical study: Dermatol surg. $2005 ; 31: 499$ - 501.

[36]. Enjolras O, Brevière GM, Roger G, Tovi M, Pellegrino B, Varotti E, Soupre V, Picard A, Leverger G : Vincristine treatment for function and life threatening infantile hemangiomas.Arch Pediatr $2004 ; 11: 99-107$.

[37]. Ezekowitz RA, Mulliken JB, Folkman J. : Interferon alfa - 2a therapy for life threatening hemangiomas of infancy N Engl J Med . 1992 : 326 : 1456-1463.

[38]. Leslie P. Lawley, Elaine Siegfried, Jane L. Todd. Propanolol treatment for hemangiomas of infancy: Risks and recommendations : Pediatr dermatol.2009; $65: 610-614$.

[39]. Jia Wei Zheng, Hua Ming Mai, Ling Zhang, Yan An Wang :Treatment or guideline for hemangiomas and vascular malformation of head and neck : Int J Clin Exp Med. 2013; 6: 377-389 